\title{
Bedeutende Neuerwerbungen Authentischer Bericht über deutsche Auswanderer
}

Eine eindrucksvolle Primärquelle über das Ergehen der deutschen Auswanderer in Amerika konnte antiquarisch erworben werden. Bei Cotta erschien 1818 ein Sammelband mit Briefen und dem Erfahrungsbericht des deutschen Dichters Moritz von Fürstenwärther (1781-1826). ${ }^{1}$ Diesen hatte sein Vetter Hans von Gagern (1766-1852) nach dem Wiener Kongress in die USA entsandt, um die Integration der deutschen Immigranten zu analysieren. Die „Instruction für den Freyherrn Moritz von Fürs-

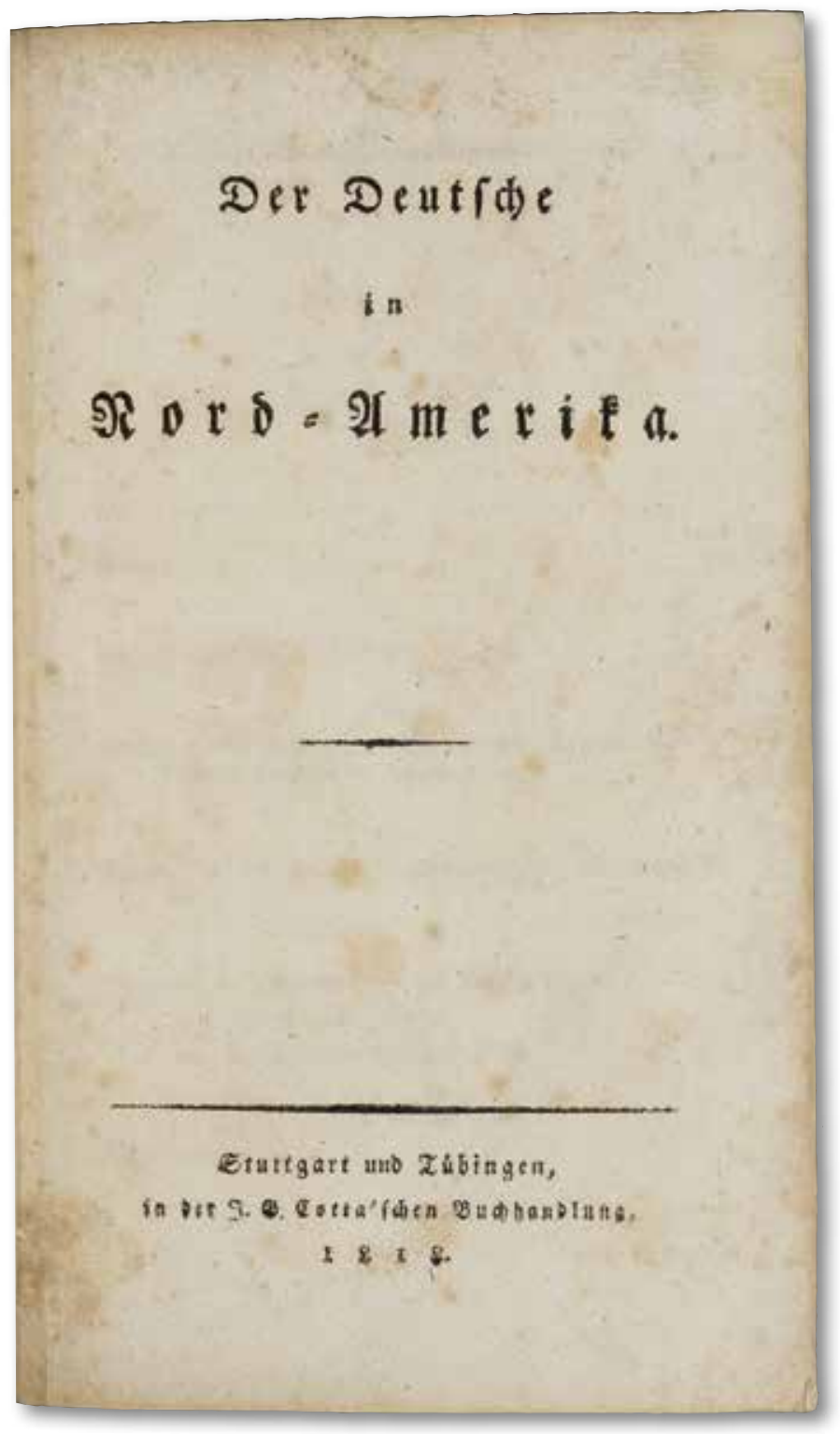

Abb. 1: Titelblatt tenwärther zum Behuf seiner Reise und Sendung nach Amerika" (17.6.1817) ist mit abgedruckt (S. 3-10). Dem nationalliberal gesinnten Diplomaten und Politiker Hans von Gagern war an authentischen Analysen gelegen. Gagern finanzierte das Projekt privat, das zur Unterrichtung der Frankfurter Bundesversammlung der Mitgliedstaaten des Deutschen Bundes gedacht war.

Auswanderungswillige Personen sollten weder zur Emigration ermutigt noch davon abgehalten werden. Für die Regierungen der deutschen Staaten waren Informationen für die Bevölkerungspolitik wichtig. Dass dieses Werk gerade im württembergischen Cotta-Verlag erschien, wurde vielleicht auch durch den hohen Anteil an Württembergern unter den Auswanderern motiviert. Gagern merkte im Nachwort zur Auswanderungswelle an: „unter uns trifft es oder traf es eben die blühendsten Länder, Würtemberg und die alte Pfalz, jenes mit der freyesten, dieses mit der gelindesten Verfassung" (S. 123). Die Verwüstungen der napoleonischen Kriege bis 1815 lagen nicht lange zurück. Der Ausbruch des indonesischen Vulkans Tambora machte 1816 zum "Jahr ohne Sommer" mit Missernten und Hunger. In Württemberg kam die eingeschränkte Religionsfreiheit infolge der rationalistischen Kirchenpolitik König Friedrichs I. hinzu, die Pietisten eine freie Glaubensentfaltung unmöglich machte. Bemühungen, wirtschaftlich leistungsstarke Bewohner im Land zu halten, führten zur Gründung Korntals 1819.

Der Bericht Fürstenwärthers lässt erahnen, wie groß die Not dieser Jahre gewesen sein muss. Sonst hätten sich Menschen nicht den lebensgefährlichen Strapazen der Seefahrt über den Atlantik ausgesetzt. So konnten bereits in den niederländischen Häfen "die Makler und Speculanten ungestraft den schändlichsten Menschenhandel treiben“ (S. 19). Von dem Kapitän eines preußischen Schiffes heißt es, dass er "sich die schändlichsten 
15

\section{Miufter eines Eontracts ber Utéerfafirt.}

$2 B$ it Enbeb Untergeidoricbene id pirain bom Sdhiff Jur einet, unb wir Fafîa giere jur andern Gcite, nefmen an, unb berpflidjten uns biemit wie Reute von Ebr.
Sapitain

Júns erfte wir Yaffagiere, unt mit obengenteibetem unfere Ricife bon bier anzuncb in Storbamerila, unb̉ mábren. zu betragen, uno mit ben bier unten gemelbeten, jwiphden bem Sapitain unb unb îbereingefonmenen Speifen bolle

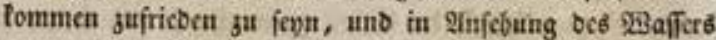

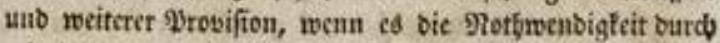
wibrigen $29 i n b$ ober fange Reife erforbert, ju idjiten nat ben शapiregeln, fo ber fiapitain noţroenbig finben wiro.

3um anberen nefmen mir an uniere gradt auf fole genbe Eontition zu bezablen:

Dic, fo in Stanoe fino felbige in Itmfterbam gu bes

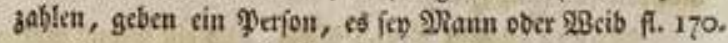

Sinber unter 4 Jafo alt, finb frey.

囚on 4 bis unter I $_{4}$ Jabren jabjlen ff. 85 .

Bon I4 Jabren, unb álter jałien fi. 170 .

Dic, io bier nidbt bezablen fonucn, unb in Simerifa

bezablen mollen, geben: es in Maun ober \$seib fi. rgo.

Sinber unter 4 Jafiren finb frep.

\$on 4 bis unter $x_{4}$ Japren jablcu fl. 95 .

\$on I4 Jafren, uno älter jablen fl. $x g$.
Abb. 2: Mustervertrag für die Überfahrt nach Amerika

Mißhandlungen gegen dieselben [die Passagiere; C.H.], unter andern einen Mißbrauch des andern Geschlechts, hatte zu Schulden kommen lassen" (S. 19f.). Die Behandlung der Auswanderer auf den Schiffen wurde als "fast durchgängig schlecht, hart, ja nicht selten unmenschlich, und empörend grausam" beschrieben (S. 37). Der Anhang zum Bericht präsentiert eine Reihe von Dokumenten und Nachrichten. Darunter findet man ein Musterformular „einer gedruckten Dienst-VerpflichtungsUrkunde oder indenture solcher, für welche der Amerikaner die Fracht und Ueberfahrt bezahlt" (Nr. XX, S. 101f.). Dies betraf solche Auswanderer, die die Kosten für die Überfahrt nicht bezahlen konnten. Einheimische Unternehmer übernahmen die Frachtgebühren und verpflichteten die Einwanderer zu einer mehrjährigen Arbeit bei schlechter Bezahlung in ihrer Firma. In einer deutsch-amerikanischen Zeitung wurde am 3.12.1817 ein Bericht mit folgender Überschrift abgedruckt: „Deutsche Sklaven am Ohio. Denn viele sehen dort so die Ablösung durch Dienst an" (S. 120).

Neben diesen abschreckenden Nachrichten führte Fürstenwärther jedoch auch viele ermutigende Aspekte aus. Die deutschen Aus- bzw. Einwanderer sollten zu einer realistischen Einschätzung gelangen: „Es ist dort kein Paradies ... Schwere Arbeit ist dort ein wesentliches Erforderniß und wohlhabend wird man nur langsam und mit Mühe"

(S. 122). Der überwiegenden Sozialstruktur der Immigranten entsprach die Nachfragesituation auf dem amerikanischen Arbeitsmarkt: „Ein Jeder, welcher nur mit einem kleinen Kapital hieher kommt, oder auch ohne das, mit Arbeit der Hände, - nicht des Kopfs - als Landmann oder Handwerker, sich ernähren will, ist gewiß, es [das Glück; C.H.] zu finden" (S. 46). Ökonomische Chancen, politische Stabilität und persönliche Freiheitsrechte motivierten in der Folge zu immer stärker zunehmenden Einwanderungswellen von Europäern: „Der allgemeine Wohlstand, welcher in diesem Lande herrscht, das Gedeihen aller menschlichen Thätigkeit, leichteres und besseres Leben bey weniger Arbeit, die Abwesenheit aller Nahrungssorgen und Besorgnisse für das Schicksal der Kinder, die daher rührende Möglichkeit früher Heirathen, wenig Abgaben, bürgerliche Freyheit, Toleranz, Sicherheit vor Revolutionen und Kriegen sind große Vorzüge desselben, und wichtige Beweggründe für alle Europäer zum Ueberzug in dasselbe" (S. 85).

Da sich dieses Buch an die verbliebene deutsche Bevölkerung richtete, wurden auch Hinweise für die "beste Zeit zur Einschiffung in Europa" gegeben: das Frühjahr "für den Landmann" und der Sommer bei "allen Handwerkern sitzender Art" (S. 89). Ein deutschstämmiger Amerikaner sei „im Allgemeinen geachtet, wegen seines Fleißes, seiner Sparsamkeit, Häuslichkeit und Redlichkeit, wegen seiner ruhigen Gemüthsart" (S. 68). Auch die Organisationsformen der Amerikaner mit deutschen Wurzeln wurden im Bericht beschrieben 
und im Anhang durch Auflistung der relevanten Institutionen konkretisiert. So gab es eine „Gesellschaft zur Ausbreitung nützlicher und erbaulicher Aufsätze in Philadelphia" (Nr. VIII, S. 95), eine "Deutsche Versorgungs- und Pflege-Gesellschaft" (Nr I., S. 92), eine "Deutsche Gesellschaft" (Nr. II, S. 92) oder eine "Jugendliche Frauenzimmer-Gesellschaft der deutschen evangelisch-lutherischen St. Michaelis- und Zions-Gemeine in Philadelphia“ (Nr. IX, S. 95).

Für gelungene Integration sprach, dass die deutschen Einwanderer schnell zu dem für Amerika charakteristischen Patriotismus beitrugen und sich als Teil der amerikanischen Staatsnation betrachteten (S. 68f.).

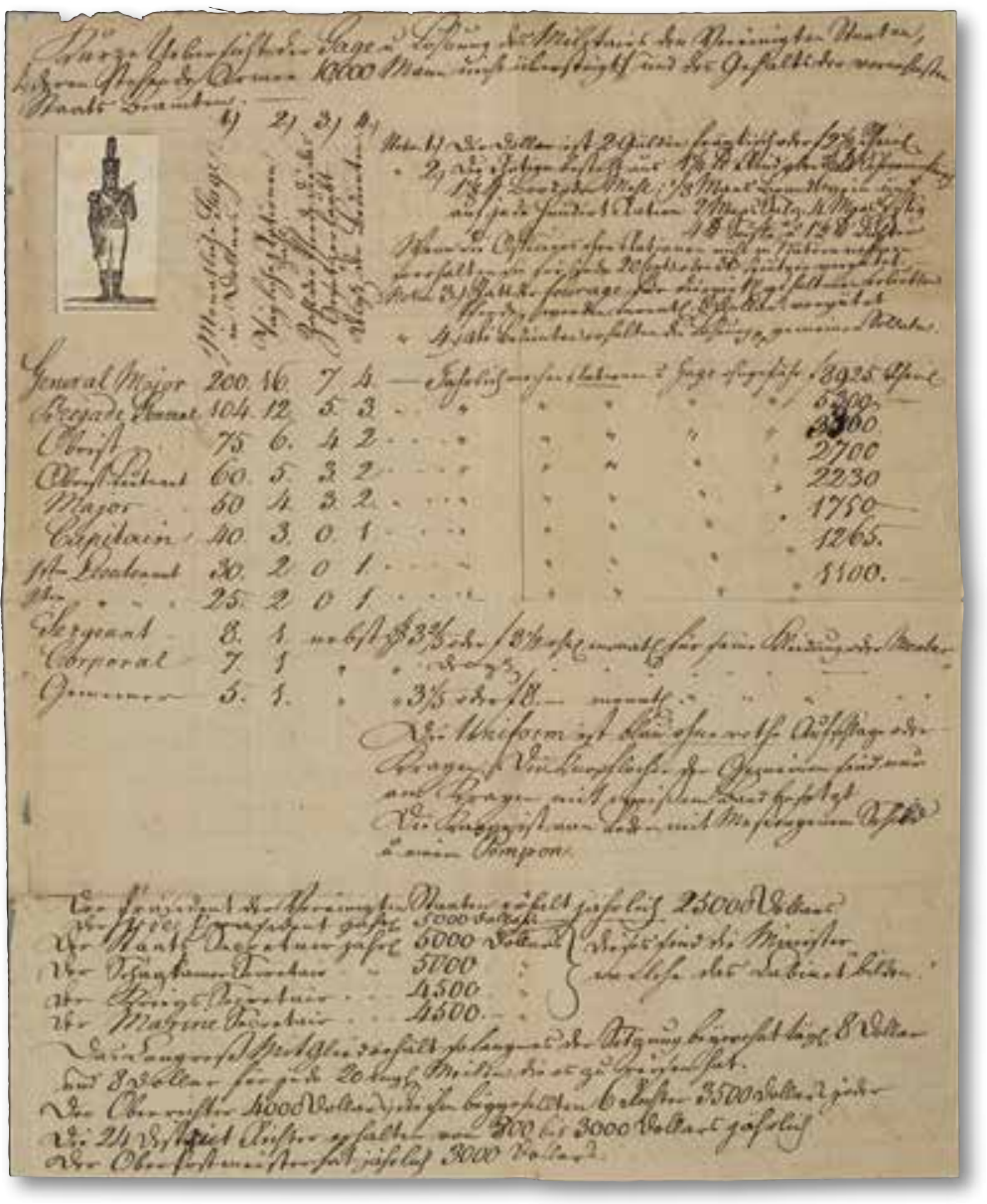

Abb. 3: Besoldungsliste der US-Armee
Zum vorliegenden Exemplar aus dem Vorbesitz des Pomologen und mit den Geistesgrößen seiner Zeit gut vernetzten Gelehrten Christian Truchseß von Wetzhausen (1755-1826) gehören zwei lose handschriftliche Dokumente. Eines davon ist eine zeitgenössische deutsche Übersetzung eines Antwortbriefes des amerikanischen Außenministers und späteren Präsidenten John Quincy Adams (1767-1848) vom 4.6.1819 an Fürstenwärther.

Darin ging Adams auf den Bericht über die deutschen Einwanderer ein und wies die Bitte um die Zuweisung einer hohen Beamtenstelle im amerikanischen Regierungsapparat an Fürstenwärther zurück. Amerika definiere sich nicht durch Privilegien, sondern gleiche Rechte. ${ }^{2}$ Das andere Dokument ist eine zeitgenössische Besoldungsliste des amerikanischen Militärs in deutscher Sprache. Es könnte von Baron von Schuler (1756-1838) stammen, der vor seiner Tätigkeit als Kammerherr am Amerikanischen Unabhängigkeitskrieg teilnahm und mit Truchseß befreundet war. ${ }^{3}$

Christian Herrmann

2) Englische und deutsche Druckfassung in: Moltmann, Günter (Hrsg.): Aufbruch nach Amerika. Friedrich List und die Auswanderung aus Baden und Württemberg 1816/17. Dokumentation einer sozialen Bewegung, Tübingen 1979, S. 241-249.

3) Vgl. Neuer Nekrolog der Deutschen, Bd. V.1 (1829), S. 5: Allgemeine Zeitung (Augsburg), 5.10.1838, S. 2102. 